\title{
Alterstice
}

Revue internationale de la recherche interculturelle

International Journal of Intercultural Research

Revista International de la Investigacion Intercultural

\section{Réimaginer la rencontre : utilisation du concept de contre-transfert culturel au retour d'une expérience d'expatriation}

\section{Francesca Bruno, Kouakou Kouassi, Marie-Rose Moro et Dominique Bernard}

Volume 6, numéro 2, 2016

URI : https://id.erudit.org/iderudit/1040632ar

DOI : https://doi.org/10.7202/1040632ar

Aller au sommaire du numéro

Éditeur(s)

Alterstice

ISSN

1923-919X (numérique)

Découvrir la revue

Citer cet article

Bruno, F., Kouassi, K., Moro, M.-R. \& Bernard, D. (2016). Réimaginer la rencontre : utilisation du concept de contre-transfert culturel au retour d'une expérience d'expatriation. Alterstice, 6(2), 59-69.

https://doi.org/10.7202/1040632ar

\section{Résumé de l'article}

Dans ce travail, l'intérêt du concept de contre-transfert culturel est mis en évidence à travers l'analyse d'une expérience d'expatriation en tant que psychologue dans une ONG locale au Cameroun. Pour cela, différents éléments du contre-transfert ont été distingués en fonction des types de rencontres : avec l'ONG d'une part, le groupe des destinataires de l'intervention d'autre part et avec chacun des participants rencontrés individuellement. L'analyse de la préparation de la mission, en matière de pré-contre-transfert, a permis d'apprécier le rôle important des motivations personnelles et d'autres éléments spécifiques. En outre, le vécu de cette expérience révèle des dynamiques transféro-contre-transférentielles liées aux mondes culturels, historiques et politiques de chacun pouvant modifier la rencontre. C'est par cette analyse approfondie que se révèle l'altérité, alors même qu'en situation d'expatriation les professionnels sont peu préparés à cette reconnaissance. En assemblant tous ces éléments, il est possible au professionnel, à son retour, d'intégrer l'intensité et la complexité d'une telle expérience dans son histoire personnelle et professionnelle et de les transmettre à tous ceux qui s'engagent dans un projet de solidarité internationale.
Droits d'auteur ( C Francesca Bruno, Kouakou Kouassi, Marie-Rose Moro et Dominique Bernard, 2016
Ce document est protégé par la loi sur le droit d'auteur. L’utilisation des services d’Érudit (y compris la reproduction) est assujettie à sa politique d'utilisation que vous pouvez consulter en ligne.

https://apropos.erudit.org/fr/usagers/politique-dutilisation/ 


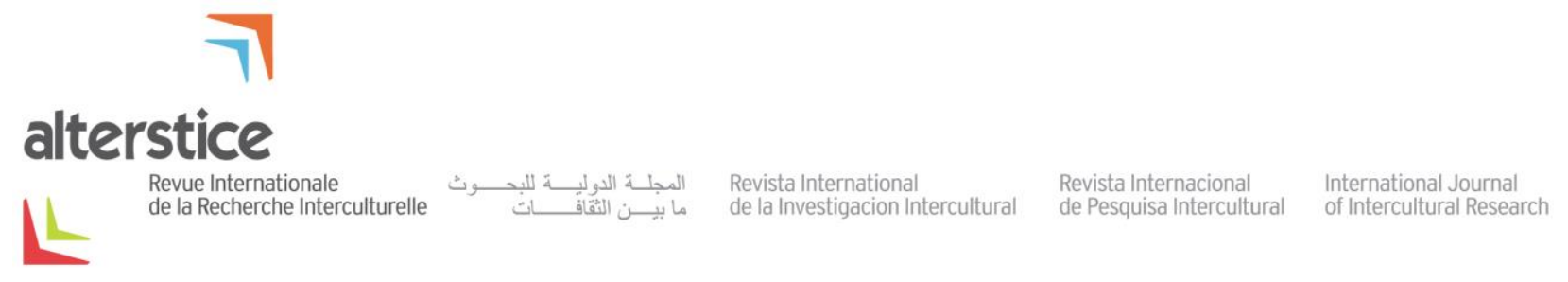

ARTICLE HORS THÈME

\title{
Réimaginer la rencontre : utilisation du concept de contre-transfert culturel au retour d'une expérience d'expatriation
}

\author{
Francesca Bruno ${ }^{1}$, Kouakou Kouassi ${ }^{2}$, Marie-Rose Moro ${ }^{1,3}$, Dominique Bernard ${ }^{4}$
}

\section{Résumé}

Dans ce travail, l'intérêt du concept de contre-transfert culturel est mis en évidence à travers l'analyse d'une expérience d'expatriation en tant que psychologue dans une ONG locale au Cameroun. Pour cela, différents éléments du contre-transfert ont été distingués en fonction des types de rencontres : avec l'ONG d'une part, le groupe des destinataires de l'intervention d'autre part et avec chacun des participants rencontrés individuellement. L'analyse de la préparation de la mission, en matière de pré-contre-transfert, a permis d'apprécier le rôle important des motivations personnelles et d'autres éléments spécifiques. En outre, le vécu de cette expérience révèle des dynamiques transféro-contre-transférentielles liées aux mondes culturels, historiques et politiques de chacun pouvant modifier la rencontre. C'est par cette analyse approfondie que se révèle l’altérité, alors même qu'en situation d'expatriation les professionnels sont peu préparés à cette reconnaissance. En assemblant tous ces éléments, il est possible au professionnel, à son retour, d'intégrer l'intensité et la complexité d'une telle expérience dans son histoire personnelle et professionnelle et de les transmettre à tous ceux qui s'engagent dans un projet de solidarité internationale.

\section{Rattachement des auteurs}

${ }^{1}$ Université Paris V Descartes, Paris, France; ${ }^{2}$ psychanalyse et ethnopsychiatrie en cabinet, Paris; ${ }^{3}$ Maison de Solenn (maison des adolescents de l'Hôpital Cochin), Paris, France; ${ }^{4}$ Centre médico-psychologique (CMP) Figuier, Hôpitaux de St Maurice, Paris, France.

\section{Correspondance}

bruno.francesca4@gmail.com

\section{Mots clés}

contre-transfert culturel; altérité; rencontres transculturelles; expatriation; transmission.

\section{Pour citer cet article}

Bruno, F. Kouassi, K., Moro, M.-R. et Bernard, D. (2016). Réimaginer la rencontre : utilisation du concept de contretransfert culturel au retour d'une expérience d'expatriation. Alterstice, 6(2), 59-70. 


\section{Introduction}

De nombreuses raisons poussent à s'engager dans la solidarité internationale, que ce soit l'aide humanitaire en urgence ou l'aide au développement pour les communautés en difficulté. Ce sont des motivations personnelles mêlées aux éléments politiques et culturels de la société d'appartenance du professionnel qui motivent l'expatriation.

Dans la dynamique de rencontre entre deux mondes, le " Nord » et le " Sud ", la mémoire, l'histoire, les enjeux de pouvoirs passés et présents s'imposent dans la rencontre, de façon plus ou moins implicite. On cherchera ici à analyser comment les niveaux individuels croisent les niveaux collectifs à travers une expérience d'expatriation au Cameroun à titre de psychologue pour un projet de soutien aux familles ayant des enfants en difficulté.

On a trouvé dans le concept du contre-transfert culturel une modalité précieuse pour révéler certains aspects de ces rencontres.

\section{Pour une compréhension du concept de contre-transfert culturel}

La notion de contre-transfert prend naissance et trouve son essence dans le cadre du processus psychanalytique et dans les dynamiques transférentielles qui lient le thérapeute et son patient.

Dans l'œuvre freudienne, le concept de contre-transfert est peu présent, souvent entouré d'ambiguïtés. Ces premières formulations, pourtant, éclairent le rôle fondamental que le contre-transfert pourrait jouer en nous dévoilant l'influence du patient sur les sentiments inconscients de l'analyste.

Ce sera à partir de la Seconde Guerre mondiale que d'autres auteurs élaboreront ultérieurement le concept selon deux courants : le premier voit le contre-transfert dans sa dimension défensive, lié aux nœuds névrotiques non élaborés de l'analyste (Racker, 1950, cité par Goyena, 2006); I'autre pense le contre-transfert comme totalité des sentiments que l'analyste éprouve envers le patient, et le considère comme un outil de compréhension de I'inconscient du patient (Heimann, 1949, cité par Denis, 2006).

La question de la définition de la spécificité du contre-transfert se complexifie avec les théorisations successives. Neyrault (1974) propose l'idée d'une précession du contre-transfert du thérapeute sur le transfert du patient : un "pré-contre-transfert ", qui se forme au moment où le thérapeute reçoit le patient et qui contient des éléments propres au thérapeute et indépendants du transfert du patient. Une utilisation plus large du concept, qui restait jusqu'à ce moment confiné à la situation de la « cure type " psychanalytique, sera possible grâce à l'extension faite par Devereux dans son travail de 1967, From anxiety to method in the behavioral sciences, traduit en français en 1980 sous le titre De l'angoisse à la méthode dans les sciences du comportement (Devereux, 1980). Dans le domaine particulier des sciences du comportement, selon Devereux, les données scientifiques peuvent éveiller de l'angoisse chez l'observateur, à laquelle il répond par une série de mouvements inconscients qui influencent la façon dont la réalité est perçue et traitée. Devereux conseille à l'observateur de ne pas chercher à diminuer cette angoisse, mais plutôt de chercher à comprendre ce qu'elle signifie : il doit se comprendre lui-même en tant que sujet-observateur.

Ainsi, Devereux (1980) définit le contre-transfert comme la somme totale des déformations de chaque observateur/chercheur/psychothérapeute, qui influence la perception et les réactions explicites et implicites, conscientes et inconscientes, envers son propre objet de recherche/patient. II montre, en outre, la présence d'une dimension culturelle dans ces réactions, due au fait que le thérapeute est aussi un homme de culture.

La notion de contre-transfert, ainsi élargie et complexifiée, est réétudiée dans un champ plus large que celui défini pour la " cure type »: le concept psychanalytique est maintenant appliqué au domaine général des sciences du comportement, car, du point de vue de Devereux, il est possible de décrire un transfert et un contre-transfert dans chaque situation où existe un échange entre une personne et une autre, à titre de cure ou de recherche. 
Le mérite de Devereux est de montrer l'existence de la dimension culturelle dans les réactions contretransférentielles, en décrivant certaines défenses émergentes face à l'angoisse : les défenses professionnelles correspondent à la façon dont les ethnologues et psychothérapeutes se réfèrent défensivement à leurs propres connaissances et méthodes (1980), l'enracinement social et culturel provoque les déformations ethnocentriques caractéristiques dues à la culture d'appartenance et enfin l'interprétation des autres et de la réalité à partir de son " modèle-de-soi » (p. 229) entraîne des déformations à se considérer soi-même comme prototype de l’humain.

En outre, il a analysé la rencontre entre l'observateur/ethnologue et le sujet observé : quand les deux viennent de cultures différentes, il ne pourra pas y avoir de réciprocité préétablie - au contraire, cette réciprocité sera à construire. Dans ce cas, les sujets d'une société tendront à se comporter avec l'étranger en conformité avec les types d'interaction courants, en poussant l'autre à assumer le statut déterminé par la " réciprocité convenable ». II s'agit d'une tendance de la partie du groupe des sujets observés à assigner à l'ethnologue/observateur un rôle précis : un "rôle complémentaire » (1980, p. 322) lorsque le professionnel, du fait de sa personnalité, adhère à ce rôle. Le chercheur/ethnologue - mais aussi le psychothérapeute - assumera et montrera, dans son contretransfert, ce rôle complémentaire, dans la mesure où il satisfait ses besoins névrotiques non résolus.

Ces conceptualisations du contre-transfert ouvrent le chemin à l'exploration ultérieure d'aspects spécifiques. La contribution de Nathan (1986) sera importante en ce sens : il définit le « contre-transfert culturel » de façon plus large comme l'ensemble des réactions d'un être humain qui rencontre un autre être humain d'une autre culture et entre en relation avec lui.

Il est important ici d'apporter une précision : dans le terme " contre-transfert culturel », il ne faut pas considérer le mot " culturel " comme un signifiant singulier car cela risque de différencier les composantes affectives et culturelles qui sont, en fait, toujours présentes et même liées dans la notion de contre-transfert culturel (Fermi, 1998).

D'autres auteurs ont développé cette notion en soulignant surtout les aspects cliniques (Comas-Diaz et Jacobsen, 1991; Moro, 1994). Moro (2004) considère le contre-transfert culturel comme un positionnement intérieur du thérapeute face à l'altérité du patient (ses manières de dire culturellement codifiées, sa manière de faire, de penser sa maladie...). Dans ses réactions à la relation (ou bien au transfert), conscientes et inconscientes, elle souligne combien la dimension affective s'articule avec les dimensions collectives (Moro, 2003).

Les implications de la prise en compte du contre-transfert dans la pratique clinique conduisent à l'élaboration de l'altérité et au processus de " décentrage culturel » (Moro, 2003, 2004). L'altérité ici s'entend comme cette qualité de ce qui est autre (Moro, 2004), qui doit être connu et reconnu comme tel. Élaborer l'altérité nécessite en tout premier lieu d'élaborer " l'altérité en soi » (Moro, 2004), cet « autre » de nous-mêmes (Kristeva, 1988). Pour Freud (1933), cette " inquiétante étrangeté » est provoquée par « quelque chose qui est, pour la vie psychique, familier de tout temps et qui lui est devenu étranger par le processus de refoulement » (p. 246). Kristeva (1988) reprend cette question comme la difficulté à se " placer par rapport à l'autre » (p. 284). En effet, lors de notre confrontation à l'autre, "l'étranger ", on découvre " notre propre étrangeté », notre propre altérité. C'est par cette voie qu'il est possible d'acquérir une position de " décentrage », disposition intérieure qui empêche de " ramener l'inconnu au connu » (Moro, 2003, p. 185).

Dans le champ spécifique de la psychiatrie humanitaire, l'hétérogénéité et les spécificités des situations imposent aux professionnels une préparation particulière aux différents types de rencontres. Très souvent, pourtant, une telle préparation se révèle inefficace sur le terrain, car il existe une perte de repères. Dans ce cas, un travail sur les contre-attitudes, sur son propre contre-transfert culturel - avec ce que celui-ci demande : élaboration de l'altérité, de l'autre en soi, du décentrage - s'avère nécessaire. 


\section{Exploration du contre-transfert culturel dans le travail d'une psychologue expatriée : rencontre avec une ONG locale et collaboration dans le projet de soutien aux familles d'enfants en difficulté}

La collaboration avec I'ONG a débuté à la suite de l'initiative personnelle de la première auteure, $\mathrm{FB}^{1}$, en tant que psychologue déjà au Cameroun dans le cadre d'un projet de Service volontaire européen. L'ONG, très active au niveau local dans l'amélioration de l'éducation, l'a invitée à prendre part à un projet appelé " Programme de psychoéducation parentale " avec l'idée de réunir les apports de la psychologie et de l'éducation spécialisée. Ce projet était conçu pour des parents ayant des enfants avec des handicaps invalidants : troubles du spectre autistique, trouble de l'attention avec hyperactivité ou difficultés d'apprentissage. Le but était d'accompagner et soutenir ces parents afin de renforcer leurs propres capacités, en stimulant leur créativité. À ce projet participaient aussi des enseignants qui souhaitaient enrichir leurs compétences face aux difficultés qu'ils pouvaient rencontrer avec ces enfants dans leurs classes.

Le projet s'est déroulé sur plusieurs journées, avec des groupes de parents et d'enseignants, sous forme de séminaires informatifs mais aussi pratiques d'ateliers et de travaux en petits groupes. Les parents étaient contactés et invités par l'ONG ou l'école de l'enfant. Ces mêmes écoles, qui faisaient toutes parties d'un réseau d'associations, « ECD Cameroon », déléguaient des enseignants.

\section{Pourquoi le contre-transfert culturel?}

«Ce qui est le plus difficile, c'est l'expatriation et la rencontre avec l'altérité si l'on n'y est pas préparé » (Moro, 2003, p. 8). C'est ainsi que la situation peut devenir « angoissante ». Mais pour sortir de cette angoisse, il est important de reconnaître ce qui agit en nous : il s'agit d'aller de l'angoisse à la méthode, comme le disait Devereux (1980). Une construction méthodologique qui « prenne en compte la situation de déséquilibre dans laquelle je me trouve, c'est-à-dire hors de mon contexte et de mes références affectives et théoriques » (Moro, 2003, p. 9).

Nous allons donc essayer de décomposer cette expérience d'expatriation à titre de psychologue en collaboration avec une ONG locale au Cameroun, pour ensuite donner un sens à la rencontre qui s'est déployée.

Nous avançons l'hypothèse que la prise en compte du contre-transfert culturel, dans ses composantes individuelles et collectives, permet d'intégrer l'expérience, d'en prendre conscience et d'en faire une transmission claire.

Nous nous s'interrogeons également sur le rôle des motivations personnelles à s'engager dans la solidarité internationale, dans le but de "comprendre certaines réactions qui échappent à l'expatrié et qui émaillent, enrichissent ou grèvent son travail » (Ouss-Ryngaert, 2008, p. 125).

Mais il n'est pas seulement question d'une histoire personnelle et professionnelle d'expatriation qui s'en trouve ainsi enrichie, c'est aussi l'histoire collective de la population locale qui entre en jeu. II s'agit donc de prendre conscience de tous ces éléments dans la rencontre. En effet, lorsque certains aspects restent inaperçus, ils peuvent contribuer à perpétuer des rapports de pouvoir asymétriques. Il est donc important de reconnaître la présence « de points aveugles, en soi et dans le cadre de la relation, pouvant contenir à notre insu des éléments excluant l'autre " (Mestre et Moro, 2012, p. 270). Cette démarche est soutenue par l'analyse du contre-transfert culturel, qui nous semble la position la plus adéquate pour acquérir et maintenir une « vigilance critique qui prenne en compte les idéologies et les rapports de pouvoir en jeu [...] » (p. 270).

Nous analyserons certaines réactions, contre-attitudes et éléments du contre-transfert culturel dans cette expérience en distinguant trois moments différents : la rencontre avec l'ONG locale, avec le groupe des parents et enseignants participants au projet, et avec les parents rencontrés individuellement. Nous analyserons les sentiments et les vécus caractérisant le moment de la préparation du projet, en les rapportant au concept de « précontre-transfert »(Neyraut, 1974). Enfin, en toile de fond, le vécu de l'expatrié sera analysé en le considérant comme un nœud important dans l'articulation du contre-transfert culturel.

\footnotetext{
${ }^{1}$ Les propos plus personnels de l'auteure sur son expérience sont présentés en couleur dans le texte, en employant le « je ».
} 
À travers des vignettes cliniques, nous présenterons les moments les plus significatifs.

Dans l'élaboration des aspects contre-transférentiels, la méthode de « triangulation » a été utilisée, soutenue par la théorie, la présence d'un superviseur, le recours à l'anthropologie et à l'histoire. Les données présentées ont été recueillies principalement par FB grâce à des prises de notes suite aux rencontres et aux réunions d'équipe.

\section{Le moment de la préparation : le "pré-contre-transfert »}

Pour décrire mon état psychique avant le début du projet avec l'ONG, je ferai référence à la prédisposition particulière du thérapeute dans l'accueil du patient, décrite par différents auteurs comme " pré-contre-transfert » ou « contre-transfert d'accueil » (Neyraut, 1974; Rouchon, 2007; Urtubey, 2006). J'ai retrouvé un vécu similaire les jours précédent le début de la mission, avec notamment des mouvements pulsionnels opposés : un allant vers l'axe du désir de découverte et l'autre allant vers la résistance à la nouveauté et le désir de rester accrochée au déjà connu.

L'aspect pulsionnel " positif » était probablement lié aux motivations qui m'avaient poussée à retourner au Cameroun et travailler avec une ONG. Après une première expérience dans le pays, restait une sorte de curiosité sur le plan professionnel : "Qu'est-ce que veut dire être psychologue ailleurs ? ". J'étais, par ailleurs, animée par la préoccupation de me rendre performante. Je cherchais des méthodes et des théories à utiliser et adapter à un contexte spécifique, sans pourtant penser à l'impact réel qu'elles pourraient avoir sur ce contexte. Je cherchais peut-être une méthodologie derrière laquelle me réfugier de façon défensive (Devereux, 1980), qui cachait une angoisse de ne pas connaître réellement ce qui m'attendait.

En outre, à ce moment-là, je ne pensais pas aux possibles points de divergence avec le travail de l'ONG ou avec les destinataires de l'action : il pouvait aussi s'agir d'un mécanisme défensif décrit par certains auteurs comme « déni des différences culturelles " (Comas-Diaz et Jacobsen, 1991). J'estimais être à l'abri des erreurs culturelles pour avoir déjà vécu six mois au Cameroun. Je croyais connaître, comprendre et accepter la culture camerounaise, sans considérer que dans le cadre d’une action différente et spécifique, les composantes culturelles pouvaient émerger encore plus fortes dans toute leur altérité.

\section{Éléments du contre-transfert culturel émergeant dans la rencontre avec l'ONG}

L'altérité que je n'avais pas prise en considération initialement a commencé à se révéler dans le travail avec l'ONG. Dès les premiers moments, j'ai découvert une façon de travailler différente, loin de la mienne, qui résonnait avec certains stéréotypes et représentations collectives de l'autre étranger. En particulier, le préjugé commun d'un peuple africain "désorganisé ", sans préparation ni bonne gestion du temps, s'est imposé dans mon imaginaire. Un exemple à ce propos :

Pendant les journées de rencontre avec le groupe de parents et d'enseignants, j'étais surprise par la façon " d'improviser » du responsable et coordinateur de I'ONG. Je ne savais jamais à l'avance ce qu'il allait aborder dans sa présentation et le découvrais sur le moment, devenant moi-même spectatrice, non sans surprise mais avec aussi un peu d'irritation.

De même, je n'étais pas informée à l'avance des dates d'activités ni des changements de programme.

Avant même de réfléchir sur le possible manque de coordination entre l'équipe de l'ONG et moi-même, mon premier réflexe a été d'interpréter ces éléments comme propres à la culture africaine.

Il m’a fallu du temps pour abandonner ce que j'avais tendance à juger comme " mal organisé » ou « mal géré » et pour comprendre et accepter ces autres rythmes, cette autre gestion du temps et de l'organisation. 


\section{Éléments du contre-transfert culturel émergeant dans la rencontre avec le groupe de parents et d'enseignants participant au projet}

Dans ces groupes, on a proposé aux participants une activité de mise en scène, pour représenter comment la famille et l'école réagissent et cherchent à s'organiser face aux difficultés exprimées par les enfants. J'ai été très impressionnée par leurs capacités à mettre en scène leur réalité culturelle permettant de faire émerger des représentations et d'entrer de façon plus approfondie dans la connaissance de l'autre. II s'agit là d'une reconnaissance de cette altérité, de cet « autre étranger » qui s'exprime à travers la créativité.

Dans une mise en scène, une famille se réunit autour du chef de famille pour discuter des comportements difficiles d'un des enfants. Le chef décide d'emmener l'enfant chez un ngengan (guérisseur devin) du village. À la suite de plusieurs traitements, la tante conseille d'aller à l'hôpital. En même temps, la mère rencontre l'enseignant de l'école et lui parle des difficultés de l'enfant. L'enseignant la rassure en lui proposant de s'en occuper en classe individualisée. II conseille aussi à la mère de refaire à la maison les exercices et jeux faits en classe. L'ensemble de ces propositions redonne ainsi espoir et courage à la famille pour prendre soin de l'enfant.

J'ai été témoin de cette transmission culturelle que je contemplais avec fascination, mais qui remettait en question mes propres théories psychologiques. Cette fenêtre sur cet autre monde faisait vaciller ma logique occidentale selon laquelle face aux difficultés comportementales d'un enfant, on a recours à l'avis d'un spécialiste, médecin ou psychologue. Je découvrais là, encore une autre façon d'être et de faire ensemble.

Les contenus théoriques et explications psychologiques que je transmettais au groupe ne trouvaient pas leur place dans ce cadre culturel et j'ai mis du temps à accepter les limites de mes théories. Une réponse s'imposait souvent à moi, face aux questions qui m'étaient adressées, celle de privilégier la création d'un espace de soin personnel, une attention individualisée. Je répondais aux questions en prenant plus de temps que nécessaire, dans une logique de soin occidentale de type individuel.

C'est grâce au travail de décentrage et de reconnaissance de l'altérité que j'ai compris et reconnu les modalités groupales du soin et de prise en charge spécifiques des cultures traditionnelles.

\section{Éléments contre-transférentiels envers les parents rencontrés individuellement}

Les rencontres avec les parents m'ont permis d'approfondir cette " altérité culturelle » et de voir émerger en moi certaines contre-attitudes devant cette autre qui se matérialisait.

Ces rencontres ont eu lieu dans le cadre de plusieurs consultations effectuées dans les écoles partenaires du réseau ECD Cameroon : l'école avait offert aux parents des enfants signalés par les enseignants la possibilité de me rencontrer.

Une caractéristique de ces consultations fut l'intérêt qu'elles ont suscité et je fus étonnée par l'affluence des parents. J'avais plutôt anticipé une réticence des parents à rencontrer une psychologue et j'ai aussitôt interprété cette affluence par le côté rassurant d’un médecin " blanc » considéré plus compétent. Il est possible qu'une telle interprétation soit la conséquence de mon jugement inconscient sur les professionnels locaux.

Par ailleurs, pendant les consultations, j'ai eu le sentiment que les parents n'étaient pas là pour se raconter mais plutôt pour avoir une réponse, une solution à leur problème. Je sentais une pression de leur part et je répondais en me défendant derrière mon rôle : j'expliquais le but des consultations, mes limites et la fonction de psychologue dans ce cadre.

Cette situation pouvait être comprise dans le cadre des cures traditionnelles : le comportement des parents correspondait à ce qui se passe pendant les consultations avec les guérisseurs ou devins. Celui qui va demander l'intervention d'un guérisseur n'a pas besoin de raconter le problème et c'est le guérisseur/devin qui se prononce sur la cause du problème et la façon de le résoudre. L'étude anthropologique d'une société peut donc venir en aide pour comprendre les dynamiques en jeu quand deux réalités culturelles se rencontrent. 
Dans d'autres types d'entretiens, j'ai rencontré des parents dans le but d'approfondir leur histoire familiale et leurs représentations autour des problèmes de leur enfant. J'ai ainsi rencontré une mère rwandaise réfugiée au Cameroun, dont le premier enfant montrait des comportements et des traits autistiques. Dans l'enquête familiale émergeaient seulement des fragments de l’histoire personnelle de la mère.

G. quitte le Rwanda vers 7 ans (elle ne peut dire l'âge exact), sans ses parents (dont on ne sait pas s'ils sont décédés), avec un groupe de réfugiés qui organise le départ pour le Zaïre. Là-bas, elle vit plusieurs années dans la région de Masisi avec d'autres déplacés. Elle raconte que son fils C., pour lequel elle consulte, n'est pas le premier, car à 15 ans, après un viol, elle fait une fausse couche. C. est aussi né d'un viol, mais elle poursuit la relation avec l'auteur du viol qui devient son conjoint. Un autre enfant naîtra, moins d'un an après. À ce moment, à cause des guerres civiles au Zaïre, G. se sépare du père des enfants et fuit en secret. Avec un petit groupe, elle vit cachée dans la forêt et entreprend le voyage pour le Cameroun. À ce moment-là, le dernier né est bébé et $\mathrm{C}$. a un an et demi. Elle dit n'avoir rien remarqué de bizarre chez $\mathrm{C}$. mais seulement qu'il ne parlait pas. Elle ne s'inquiétait pas encore. Les femmes qui vivaient avec elle la rassuraient en disant qu'il pouvait s'agir d'un simple retard. Maintenant elle est au Cameroun depuis environ 4 ans où elle a rencontré un homme, rwandais, avec qui elle a eu un troisième enfant.

L'image d'une très jeune fille qui vit seule pendant plus de 15 ans dans l'incertitude et la terreur, victime de deux viols, dont l'agresseur devient son conjoint : tout cela touchait mon identité féminine occidentale et bouleversait mes idéologies personnelles et celles de ma société d'appartenance. J'étais impressionnée par l'absence d'émotivité et la difficulté à préciser des détails, comme s'il s'agissait d'une histoire maintes fois racontée. J'avais la sensation d'un détachement émotionnel qui provoquait également de la sidération en moi. Je n’ai pas pu poser de questions pour approfondir certains aspects, je n'ai pas pu stimuler l'émergence d'émotions et de sentiments liés à ce récit qui restait ainsi « nu » de sens. Ce récit devenait quelque chose de traumatique et déclenchait en moi pitié et stupéfaction.

Dans ces situations, comme l'explique Lachal (2006), le récit traumatique provoque chez le clinicien un " scénario émergent ", résultat des capacités créatives du thérapeute dans un moment d'empathie avec le patient (Quattroni et Mestre, 2008). À partir de ce scénario émergent, le thérapeute peut transformer les images virtuelles en mots dans un récit qui pourra réanimer les capacités narratives du patient.

Le scénario que j'ai eu en réponse à ce récit fut : une forêt noire et une femme qui marchait avec ses deux enfants sur le dos et dans les bras, pendant des heures sans mot dire. Mais je n'ai pas réussi à transformer ce scénario en mots. Les capacités narratives de G. restaient pauvres et moi, je restais dans la sidération du traumatisme qui m'était transmis. Dans cette situation, je me cachais derrière l'objectif que j'avais fixé pour l'entretien, évitant de laisser trop d'espace à cette histoire traumatique. Le cadre de la rencontre restait donc rigide, suggérant une utilisation défensive du cadre, qui empêchait l'intégration de ces éléments.

"Que faire d'une telle rencontre avec le trauma? ». Cette question n'est survenue que dans l'après-coup, au cours de mes réflexions et de l'élaboration du contre-transfert.

\section{La toile de fond : éléments contre-transférentiels présents dans toutes ces rencontres}

À côté des aspects contre-transférentiels décrits plus haut, une sensation particulière m’a accompagnée durant toute la mission. Une image symbolise ce vécu : pendant les journées d'activité avec le groupe des participants, l'équipe de l'ONG me préparait une table et une chaise devant les participants, ce qui me mettait mal à l'aise. Cette table et cette chaise, disposées pour moi, représentaient la place que l’ONG me réservait et m'avait assignée dès le début. Ce rôle me confinait dans celui du "docteur blanc détenteur de savoir ». Je me sentais ainsi investie de pouvoirs particuliers par le fait d'avoir été formée en Europe. La table et la chaise m'évoquaient un rapport d'une asymétrie évidente où moi la " blanche " avait une place supérieure, rappelant un nouveau rapport colonisateurcolonisé. Ces traces du colonialisme se retrouvent souvent dans les relations entre occidentaux et peuples africains et l'histoire du Cameroun, comme bien d'autres, est imprégnée de cette modalité relationnelle entre blancs et noirs en terme de dominant-dominé. 
Ainsi, ma réponse à la gêne éprouvée dans cette situation était instinctive et physique : j'essayais de déplacer la chaise à côté de la table, je restais debout en marchant devant la table. Mais il y avait aussi de l'ambivalence, d'un côté l'adhésion à ce rôle et de l'autre un mal-être à me trouver dans une telle position de " docteur blanc/colonisateur $"$.

\section{Poser l’action, agir la réflexion : ré-imaginer la rencontre}

Le professionnel "psy » qui décide de s'engager dans des projets de solidarité internationale se retrouve à vivre des expériences si singulières qu'elles peuvent être source d'angoisse. En effet, se retrouver en dehors de son propre contexte social, culturel, linguistique et professionnel, se retrouver face à une diversité qu'on ne sait pas pénétrer, rencontrer cet "inquiétant étranger ", qui résonne avec ce non-connu de nous-mêmes, ce refoulé provoque une déstabilisation.

Ainsi, du fait de cette angoisse et des mouvements défensifs, il se peut que la mission se déroule sans pouvoir prendre en compte l'altérité et vivre la richesse de la rencontre. C'est le risque de partir sans assez de " curiosité ", comme le pense Moro (2003) : la curiosité nécessaire qui nous pousse à traverser nos frontières car on croit pouvoir trouver quelque chose de précieux et de singulier qui peut nous transformer et nous ouvrir à de nouveaux systèmes de pensée, à « un nouvel usage du monde possible » (Bouvier, 2001, cité par Moro, 2003). Ainsi, celui qui rentre devrait chercher à transmettre quelque chose de ce qu'il a appris, et ainsi aider à préparer ceux qui suivent à cette notion de curiosité et à la beauté de ces rencontres (Moro, 2003, p. 10).

Ce qui a été vécu au retour, ce fut l'impossibilité de transmettre et témoigner des actions entreprises. Prise entre sidération et fascination, je m'interrogeais sur mon rôle pour me rendre compte qu'il n'avait pas été clair et que la désorientation prévalait.

C'est dans cette prise de conscience et dans l'analyse de mon contre-transfert que peu à peu les choses se sont révélées. "Lorsque nous n'apprenons plus rien de notre rencontre avec l'autre, il est grand temps de revoir nos positions contre-transférentielles, donc par là même, nos concepts, théories et méthodes " (Rouchon, 2007, p. 193).

Dans mon expérience, j'ai pu mettre en évidence ma difficulté à me préparer à la rencontre, à me situer dans la relation à l'autre, investi alors de manière ambivalente entre « fascination et angoisse » (Ouss- Ryngaert, 2003).

De plus, la façon de travailler des membres de l'ONG me paraissait très différente de la mienne et résonnait avec certaines représentations collectives de l'autre « étranger ».

Dans les rencontres avec les destinataires du projet aussi, un monde différent s'ouvrait à moi et renforçait mon questionnement sur la manière dont les communautés s'organisent face aux difficultés d'un de leurs membres, dont les membres d'une même famille s'engagent dans une dynamique de cure, dont les modalités de soin traditionnelles sont mises en place.

Tous ces éléments caractéristiques de certaines sociétés traditionnelles doivent être non seulement connues et acceptés, mais aussi intégrés dans nos actions pour que le psychologue expatrié en mission ne se retrouve pas seul à travailler avec ses outils et son cadre rigide, mais au contraire puisse faire preuve de malléabilité et souplesse dans l'interaction avec la réalité locale.

La tendance qu'on a vue émerger à cette occasion est le recours aux défenses particulières, appelées par Devereux " défenses professionnelles » (1980). Les positions méthodologiques et les dispositifs techniques peuvent être utilisés d'abord comme des défenses qui déforment et qui interfèrent de multiples façons avec ce qui se passe dans la rencontre. Effectivement, dans cette expérience, le recours à " nos » méthodologies et théories occidentales de la psychologie a trouvé ses limites dans cette clinique, qui nous rappelle que les bases psychologiques et psychiatriques intègrent des présupposés culturels, ici non reconnus. 
Devereux (1980), souligne aussi que, devant des matériaux traumatisants, le spécialiste du comportement apprend à utiliser sa position professionnelle comme un moyen de réduire l'angoisse. C'était le cas avec $\mathrm{M}^{\mathrm{me}} \mathrm{G}$. présenté plus haut, où dans la rencontre avec le traumatisme d'une jeune mère, je me suis défendue derrière le cadre rigide fixé pour ces rencontres et qui n’a pas permis d'intégrer ce matériel clinique, en laissant les deux protagonistes dans un vide émotionnel.

Le contre-transfert, même dans ses dimensions culturelles, est d'abord une expérience singulière pour la personne, mais certaines de ces réactions sont partagées par la plupart des membres de sa société car elles dérivent des aspects spécifiques du contexte culturel. Ce sont donc ces éléments consensuels qui pourront être informatifs à l'échelle collective (Rouchon, Reyre et Taieb, 2009). Dans la mesure où le contre-transfert culturel dépend aussi de la manière dont l'altérité culturelle est traitée dans notre société, on pourra peut-être gagner quelque chose si on cherche à élaborer cette altérité collectivement. La notion de contre-transfert culturel, en fait, se trouve au carrefour de l'individuel et du collectif (Moro, 2004), et pour cela pose la question de l'élaboration de l'altérité au niveau également collectif.

Dans les propositions pour un positionnement du travail en psychiatrie humanitaire, Ouss-Ryngaert (2003) nous invite à « retrouver en soi la familiarité de ce que l'autre nous fait vivre et prendre en compte l'autre de soi-même, l'autre étranger, l'autre du trauma " (p. 103). II s'agit d'une expérience de déséquilibre, car il fait vaciller nos propres fondements. Cependant, on pourra y trouver un énorme enrichissement si, en acceptant ces autres, on " ouvre le dialogue fécond qui permet la rencontre entre soi, son expérience, sa culture et celles de l'autre " (p. 104).

Ainsi, dans l'aspect contre-transférentiel lié au vécu du rôle assigné de " docteur blanc détenteur du savoir », il est possible d'identifier la dynamique qui croise les dimensions individuelles et collectives. J'ai rapproché la sensation d'avoir eu ce rôle assigné par l'ONG et par les autres participants au "rôle complémentaire " proposé par Devereux (1980). II est probable que l'ONG et la communauté m'aient investie comme le spécialiste auquel on n'a pas accès et en même temps symbole du « blanc colonisateur » que ma figure incarnait.

De mon côté, j'étais prise par ces dynamiques transféro-contre-transférentielles pour plusieurs raisons : aucune ONG ne « m'envoyait » et cela m'engageait à m'adapter au plus près des besoins de la mission qui m'accueillait. En outre, dans mon pays, je n'avais pas encore de statut social et professionnel affirmé comme psychologue. Enfin, je n'avais pas reçu de formation spécifique, mais j'étais seulement animée par des questionnements comme " que veut dire être psychologue ailleurs? ", liés au désir de chercher une autre voie de formation et de professionnalisation, trop long et trop classique dans mon pays. Finalement, je n'ai pas pu trouver " ailleurs " le rôle que je n'avais pas construit en moi.

Si on ne prend pas en compte tout cela, le risque est d'alimenter des dynamiques relationnelles où chacun met en place ses imagos sans qu'il y ait de vraie rencontre entre les deux mondes. Dans ma situation, je n'ai pas réalisé qu'en habitant le rôle complémentaire, je contribuais à un type de relation de pouvoir asymétrique en faveur du "blanc ". C'est le risque pour tous les programmes de coopération ou d'aide internationale "Nord-Sud ", où le Nord agit en restant au Nord et le Sud restant toujours au Sud, sans rencontre possible.

On pourrait le dire autrement, en reprenant les paroles de Fanon (1952) : «le blanc est enfermé dans sa blancheur et le noir enfermé dans sa noirceur " (p. 31). Il nous montre, en effet, comment la colonisation fonctionnait en décrivant l'intériorisation par le " noir » d'un complexe d'infériorité et par le " blanc colonisateur » d'un complexe de supériorité. Ces différents aspects relationnels, cette opposition structurelle entre colonisateur et colonisé, entre blanc et noir, réapparaissent ainsi et nous révèlent le caractère souvent inachevé de la décolonisation.

En fait, si la barrière " entre le maître et l'esclave est aujourd'hui abolie, nos inconscients en gardent la trace ", souligne Kristeva (1988), et les représentations collectives véhiculées par nos sociétés (Fernando, 1995) portent les fantasmes de cet imaginaire colonial (Hall, 1997, cité par Sturm, 2006). 
Ainsi, le contexte socio-historique d'appartenance de deux individus et l'inscription de l'Histoire dans la mémoire collective peuvent mobiliser nombre d'affects : le travail sur cette multiplicité d'éléments, individuels et collectifs, est nécessaire pour que tout ce qui se joue dans une rencontre trouve sa place. C'est ce qui permet de construire le « décentrage culturel », lequel semble effectivement plus facile à acquérir à travers " l'expatriation de soi-même et la pratique clinique en situation d'expatriation » (Moro, 2004).

On pourrait ainsi envisager la construction, dans ce type de projet ou d'aide au développement, d'un dispositif spécifique où le contre-transfert culturel de tous les acteurs puisse être reconnu, identifié, élaboré. Ce dispositif pourrait s'inspirer de la consultation d'ethnopsychiatrie de l'hôpital d'Avicenne (Seine-Saint-Denis, France), c'est-àdire un dispositif spécifique à "géométrie variable " (Moro, 2004 p. 99), où la diversité d'origine et de langues parlées dans le groupe figure l'altérité (Moro, 2004, p. 114) mais participe également à la transformation de cette altérité en "levier thérapeutique » (Moro et Nathan, 1990). C'est-à-dire que ces "parcelles» d'altérité matérialisées par les membres du groupe et représentées dans l'espace permettent « d'expérimenter une autre forme d'altérité qui ne soit ni monstrueuse ni menaçante ni destructrice, mais au contraire une altérité figurable et créatrice » (Moro, 2004, p. 114).

De nouvelles perspectives de recherche sont nécessaires pour adapter un tel dispositif dans le cadre des missions humanitaires où des "psy" expatriés collaborent avec des professionnels locaux. Certains programmes d'intervention médico-psychologiques de Médecins sans Frontière ont d'ailleurs déjà développé des formes de supervision sur le terrain, et des possibilités de témoignage au retour (Lachal, Ouss-Ryngaert et Moro, 2003). Si ces expériences sont très riches, il nous semble important d'insister sur la nécessité de prendre en compte la spécificité d'une situation et de penser à une co-construction sur le terrain : ainsi pourra se révéler la complexité des contacts interculturels à la fois au niveau intrapsychique, intersubjectif, inter-groupal et interinstitutionnel.

En conclusion, l'élaboration et la prise en compte du contre-transfert, dans toute pratique clinique, nous permet de nous insérer sur le parcours de l'élaboration de l'altérité en soi, de l'altérité de l'étranger : ainsi on pourra effectivement trouver la position la plus favorable qui nous prépare à accueillir l'autre et à vivre pleinement la valeur de la rencontre.

\section{Références bibliographiques}

Comas-Diaz, L. et Jacobsen, F. (1991). Ethnocultural transference and countertransference in the therapeutic dyad. American Journal of Orthopsychiatry, 61(3), 392-402.

Denis, P. (2006). Incontournable contre-transfert. Revue française de psychanalyse, 70(2), 331-350.

Devereux, G. (1980). De l'angoisse à la méthode dans les sciences du comportement (traduit par H. Sinacoeur). Paris : Flammarion. (Ouvrage original publié en 1967 sous le titre From anxiety to method in the behavioral science).

Fanon, F. (1952). Peau noire, masques blancs. Paris : Seuil.

Fermi, P. (1998). Contre-transfert culturel? Communication présentée à Cliniques entre deux rives, Université de Bordeaux-2. http://perso.orange.fr/geza.roheim/html/contrans.htm

Fernando, S. (1995). Mental health in a multi-ethnic society. A multi-disciplinary handbook. Londres : Routledge.

Freud, S. (1933). L'inquiétante étrangeté. Essais de psychanalyse appliquée (traduit par M. Bonaparte et E. Marty). Paris : Gallimard. (Ouvrage original publié en 1919 sous le titre Das Unheimliche).

Goyena, A. (2006). Heinrich Racker : le contre-transfert comme nouveau départ de la technique psychanalytique. Revue française de psychanalyse, 70(2), 351-370.

Kristeva, J. (1988). Étrangers à nous-mêmes. Paris : Fayard.

Lachal, C. (2006). Le partage du traumatisme, contre-transferts avec les patients traumatisés. Grenoble : La pensée sauvage.

Alterstice - Revue Internationale de la Recherche Interculturelle, vol. $6, n^{\circ} 2$ 
Lachal, C., Ouss-Ryngaert, L. et Moro, M. (dir.) (2003). Comprendre et soigner le trauma en situation humanitaire. Paris : Dunod.

Mestre, C. et Moro, M. (2012). L'intime et le politique. Projet pour une ethnopsychanalyse critique. L'autre, cliniques, cultures et sociétés, 13(3), 263-272.

Moro, M. (1994). Parents en exil. Psychopathologie et migrations. Paris : Presses universitaires de France.

Moro, M. (2003). Penser la psychiatrie humanitaire. Dans C. Lachal, L. Ouss-Ryngaert et M. Moro (dir.), Comprendre et soigner le trauma en situation humanitaire (p. 3-26). Paris : Dunod.

Moro, M. (2004). Psychiatrie transculturelle de l'enfant et de l'adolescent. Paris : Dunod.

Moro, M. et Nathan, T. (1990). Clinique de la rencontre. Présentations de quelques principes techniques de l'ethnopsychiatrie. Psychologie et éducation, 3(4), 23-36.

Nathan, T. (1986). La folie des autres. Traité d'ethnopsychiatrie générale. Paris : Dunod.

Neyraut, M. (1974). Le transfert. Paris : Presses universitaires de France.

Ouss-Ryngaert, L. (2003). Être acteur du soin psychique en situation humanitaire. Dans C. Lachal, L. Ouss-Ryngaert et M. Moro (dir.), Comprendre et soigner le trauma en situation humanitaire (p. 89-106). Paris : Dunod.

Ouss-Ryngaert, L. (2008). Relations amoureuses en terrain humanitaire. L'autre, cliniques, cultures et sociétés, 9(1), 119-128.

Quattroni, B. et Mestre, C. (2008). Table ronde : migrations, enfance et familles : altérité et diversité culturelles. Contre-transfert et scénario émergent dans les psychothérapies de mères traumatisées : à propos d'un cas. Neuropsychiatrie de l'enfance et de l'adolescence, 56, 206-210.

Rouchon, J.-F. (2007). La notion de contre-transfert culturel: enjeux théoriques, cliniques et thérapeutiques (Thèse de doctorat). Université de Nantes. En ligne à : www.clinique-transculturelle.org

Rouchon, J.-F., Reyre, A. et Taieb, O. (2009). L'utilisation de la notion de contre-transfert culturel en clinique. L'autre, cliniques, cultures et sociétés, 10(1), 80-89.

Sturm, G. (2006). Le racisme et l'exclusion. Dans M. Moro, Q. De la Noë et Y. Mouchenik (dir.), Manuel de psychiatrie transculturelle, travail clinique, travail social (p. 197-216). Grenoble : La pensée sauvage.

Urtubey, L. (2006). Des origines du contre-transfert. La revue de psychanalyse, 70(2), 371-384. 